An Investigation Into Bayesian Networks for Modeling National Ignition Facility Capsule Implosions

J. Mitrani

August 21, 2008 
This document was prepared as an account of work sponsored by an agency of the United States government. Neither the United States government nor Lawrence Livermore National Security, LLC, nor any of their employees makes any warranty, expressed or implied, or assumes any legal liability or responsibility for the accuracy, completeness, or usefulness of any information, apparatus, product, or process disclosed, or represents that its use would not infringe privately owned rights. Reference herein to any specific commercial product, process, or service by trade name, trademark, manufacturer, or otherwise does not necessarily constitute or imply its endorsement, recommendation, or favoring by the United States government or Lawrence Livermore National Security, LLC. The views and opinions of authors expressed herein do not necessarily state or reflect those of the United States government or Lawrence Livermore National Security, LLC, and shall not be used for advertising or product endorsement purposes.

This work performed under the auspices of the U.S. Department of Energy by Lawrence Livermore National Laboratory under Contract DE-AC52-07NA27344. 


\title{
An Investigation Into Bayesian Networks for Modeling National Ignition Facility Capsule Implosions
}

\author{
James Mitrani
}

August 14, 2008

\section{Abstract}

Bayesian networks (BN) are an excellent tool for modeling uncertainties in systems with several interdependent variables. $A B N$ is a directed acyclic graph, and consists of a structure, or the set of directional links between variables that depend on other variables, and conditional probabilities (CP) for each variable. In this project, we apply BN's to understand uncertainties in NIF ignition experiments. One can represent various physical properties of National Ignition Facility (NIF) capsule implosions as variables in a BN. A dataset containing simulations of NIF capsule implosions was provided. The dataset was generated from a radiation hydrodynamics code, and it contained 120 simulations of 16 variables. Relevant knowledge about the physics of NIF capsule implosions and greedy search algorithms were used to search for hypothetical structures for a BN. Our preliminary results found 6 links between variables in the dataset. However, we thought there should have been more links between the dataset variables based on the physics of NIF capsule implosions. Important reasons for the paucity of links are the relatively small size of the dataset, and the sampling of the values for dataset variables. Another factor that might have caused the paucity of links is the fact that in the dataset, $20 \%$ of the simulations represented successful fusion, and $80 \%$ didn't, (simulations of unsuccessful fusion are useful for measuring certain diagnostics) which skewed the distributions of several variables, and possibly reduced the number of links. Nevertheless, by illustrating the interdependencies and conditional probabilities of several parameters and diagnostics, an accurate and complete BN built from an 
appropriate simulation set would provide uncertainty quantification for NIF capsule implosions.

\section{An Introduction to Bayesian Networks}

\subsection{The Bayesian Approach to Probability}

An essential tool used in every field of the sciences is probability. Without it, there would be practically no advances in many fields of science. The traditional approach towards probability, or the frequentist approach [1][2], involves studying an event by performing a large number of trials, and measuring the frequency of successful outcomes. A larger number of trials will lead to a more accurate estimate of the probability of success for this event. This approach is ubiquitous, and is used for measuring events such as mutation rates in DNA replication, to the viscosity of liquids, to the work functions of metals.

However, some events don't lend themselves to a large number of trials because the event might occur very rarely (such as measuring certain astrophysical properties), or because performing a single trial is very expensive (such as testing nuclear weapons). Studying these events with the aforementioned frequentist approach is very difficult. A different approach towards studying these situations is called the subjective approach [2], and involves using Bayesian statistics. Bayesian statistics are more general than frequentist statistics since Bayesian statistics regards probability as a degree of belief [1], which removes the need for the large number of trials necessary for frequentist statistics. In probability, the product rule states

$$
P(X \mid Y)=\frac{P(X, Y)}{P(Y)},
$$

where $P(X \mid Y)$ represents the probability that event $\mathrm{X}$ is true given that event $\mathrm{Y}$ is already true, $P(X, Y)$ represents the probability that both events $\mathrm{X}$ and $\mathrm{Y}$ are true, and $P(Y)$ represents the probability that event $\mathrm{Y}$ is true. (Intuitively, this rule states that the probability for any two events to occur is the probability that the first event and the probability that the second event occurs given that the first event has already occurred.) By noting that $P(X, Y)=P(Y, X)$, one can drive Bayes' theorem, which states that

$$
P(X \mid Y)=\frac{P(Y \mid X) \cdot P(X)}{P(Y)}
$$

As stated previously, Bayes' theorem is practical for studying events that don't lend themselves to a large number of studies. For example, suppose one has a hypothesis 
about some astrophysical properties. Then, when those properties can be measured and data about those properties can be recorded, Bayes' theorem states that

$$
P(H \mid D)=\frac{P(D \mid H) \cdot P(H)}{P(D)},
$$

where $\mathrm{P}(\mathrm{H} \mid \mathrm{D})$ is the probability that the hypothesis is true given that the data was observed, and $\mathrm{P}(\mathrm{D} \mid \mathrm{H})$ is the probability that the data will be observed given that the hypothesis is accurate. Alternatively, $\mathrm{P}(\mathrm{H})$ is the prior probabliity of the hypothesis being correct, $\mathrm{P}(\mathrm{H} \mid \mathrm{D})$ is the posterior probability of the hypothesis being correct, and $\mathrm{P}(\mathrm{D} \mid \mathrm{H})$ is the probability of observing the data given the specific hypothesis. For example, assume a scientist is deciding between two different models regarding those properties, and according to the scientist's prior studies, the first hypothesis $\left(\mathrm{H}_{1}\right)$ is accurate with probability $\mathrm{p}_{1}$, and the second hypothesis $\left(\mathrm{H}_{2}\right)$ is accurate with probability $\mathrm{p}_{2}$. Then, one can determine the relative likelihood of the two hypotheses with the ratio of the probabilities of each hypothesis being true given the data observed, or

$$
\frac{P\left(H_{1} \mid D\right)}{P\left(H_{2} \mid D\right)}=\frac{P\left(D \mid H_{1}\right) \cdot P\left(H_{1}\right)}{P\left(D \mid H_{2}\right) \cdot P\left(H_{2}\right)}=\frac{P\left(D \mid H_{1}\right) \cdot p_{1}}{P\left(D \mid H_{2}\right) \cdot p_{2}},
$$

where the P(D) term from Eq. 3 gets canceled. Since all terms in Eq. 4 are known, the scientist can determine the relative likelihood of the two hypotheses.

\subsection{Bayesian Networks}

As mentioned previously, Bayes' theorem takes into account how probabilities change when data is observed. However, studying a system with a large number of events or variables can be very complicated. An excellent tool to study these systems is a Bayesian network (Bayes net, or BN). A BN is a directed acyclic graph (DAG), meaning it contains no feedback mechanisms (Graph IV in Fig. 1) [2][3]. In a BN, the variables are called nodes and are represented by circles, and the interdependencies are called edges and are represented by arrows. The arrow pointing from $\mathrm{A}$
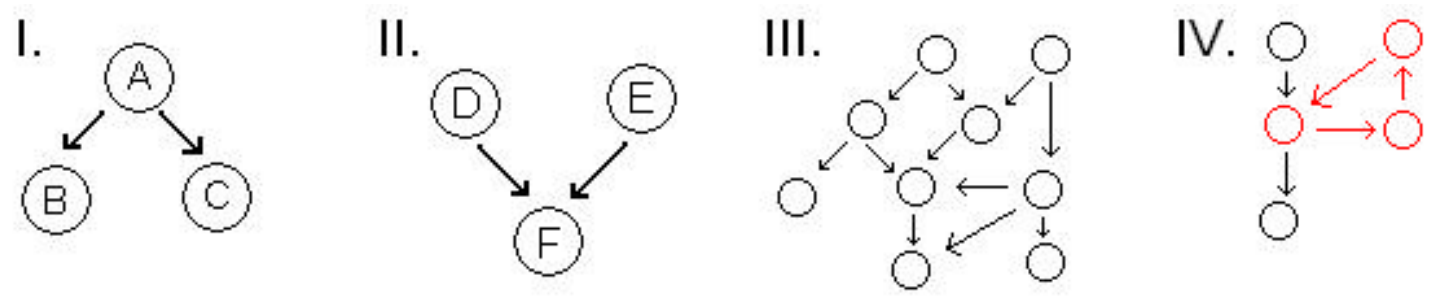

Figure 1: Graphs I-III are DAG's, and represent Bayesian networks (BN's). Graph IV is not a $\mathrm{BN}$, as it contains a feedback mechanism (red). 
to B (Graph I. in Fig. 1) means that event B directly depends on event A, and not vice versa. In Graph I., A is said to be the parent of B and C. In Graph II., both $\mathrm{D}$ and $\mathrm{E}$. are the parents of $\mathrm{F}$.

BN's allow for visualization of the interdependencies between events. A major advantage is that BN's simplify our calculation of the joint probability distribution (joint PDF) of the system, or the probability that the system is in a specific state. With the joint probability of the system, we can calculate any conditional probability, which is extremely useful for measuring systems with limited data. Because each event only depends on its parents, the probability of each event can be calculated by[2]

$$
P\left(X=x_{i}\right)=P\left(X=x_{i} \mid \text { Parents }\left(X=x_{i}\right)\right)
$$

where $\mathrm{P}\left(\mathrm{X}=\mathrm{x}_{i}\right)$ is the probability that event $\mathrm{X}$ is in a specific state. If event $\mathrm{X}$ were discrete, then $\mathrm{P}\left(\mathrm{X}=\mathrm{x}_{i}\right)$ would be the probability that $\mathrm{X}$ is in state $\mathrm{x}_{i}$. If $\mathrm{X}$ were continuous, then $\mathrm{P}\left(\mathrm{X}=\mathrm{x}_{i}\right)$ would be the probability that the value of $\mathrm{X}$ lies between $\mathrm{x}_{i}$ and $\mathrm{x}_{i}+\Delta \mathrm{x}$. Although BN's can theoretically deal with events with a continuous distribution of values, one usually needs a distribution function to model those values [4]. Therefore, the rest of this paper will discuss BN's with discrete valued variables.

\subsection{A Simple Example of Bayesian Networks}

An example of modeling a system with BN's concerns the case of a patient who has tested postive for a rare, harmful disease. Treatment for this disease is expensive and would severely reduce her quality of life, so it's important that she receive accuate information about whether or not she has the disease. The disease is rare, and only occurs in one in a thousand people. The patient tests positive $99.5 \%$ of the time if she has the disease, and $10 \%$ of the time if she is healthy (Fig. 2A). Initally, one might think that a patient who tests positive almost certainly has the disease. However, from Bayes' theorem (Eq. 2), we find out the patient's probability

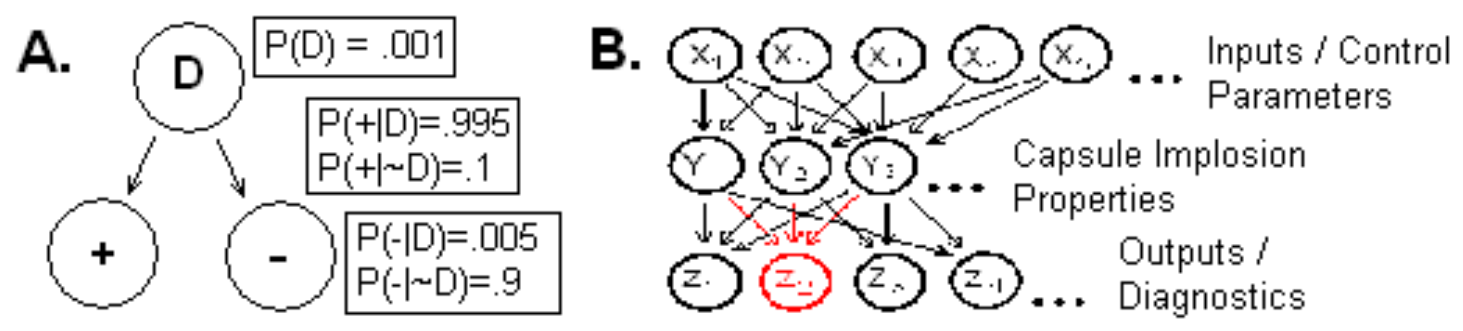

Figure 2: The first graph (left) shows a Bayesian net for the rare disease example, illustrated below. The second graph (right) shows a hypothetical Bayes net for a NIF capsule implosion. 
of having the rare disease is

$$
P(D \mid+)=\frac{P(+\mid D) \cdot P(D)}{P(+)}=\frac{.995 \cdot .001}{.995 \cdot .001+.1 \cdot .999}=9.86 \cdot 10^{-3},
$$

which is much lower then one would initially expect. An intuitive explanation of the solution is that the cause of a patient's positive test is much more likely to come from a false positive reading than from actually having the disease, because the prior probability of having the disease is so rare.

Although this problem can be solved without its Bayes net (Fig. 2A), the Bayes net does illustrate the concept of conditional independence. From this example, the correlation of patients testing positive and testing negative is very close to -1, as it's extremely rare (though not impossible) for a patient to receive positive and negative test results. However, because both the probabilities of positive and negative test results only depend on whether the patient has the disease, the events of a positive test result and a negative test result are conditionally independent from each other. Formally, this can be expressed as

$$
P(+\mid D,-)=P(+\mid D),
$$

where $\mathrm{P}(+\mid \mathrm{D},-)$ is the probability that the patient tests positive given that we know whether the patient has the disease and whether the patient tests negative, and $\mathrm{P}(+\mid \mathrm{D})$ is the probability that the patient tests positive given that we know whether the patient has the disease. (If events $\mathrm{X}$ and $\mathrm{Y}$ are truly independent, then $\mathrm{P}(\mathrm{X} \mid \mathrm{Y}$ ) $=\mathrm{P}(\mathrm{X})$.)

The goal of this project is to model the probabilistic interdependencies of NIF capsule implosions with Bayes nets. Section 2 will present various methods of generating a Bayesian network structure from a dataset (referred to as the 'dataset' throughout this paper) of simulations of NIF capsule implosions (provided by Brian Spears, Lawrence Livermore National Laboratories, Livermore, CA) generated by a radiation hydrodynamics code [5]. The dataset contained 120 simulations with 16 variables (Appendix). The end of section 2 will describe estimating the conditional probabilities for the Bayes net. Section 3 will present the results from generating a Bayes net based on the dataset. Finally, section 4 will present a brief conclusion, including future considerations. 


\section{Training an optimal Bayesian Network Based on Raw Data}

\subsection{Finding an Optimal Subset of Structures for the Bayesian Network}

All calculations were perfomed with Matlab (R2006b), the Bayes Net Toolbox for Matlab [6], and the Structure Learning extension [7].

As stated previously, since we were interested in a Bayes net with discrete valued variables, we had to discretize the data in the dataset. We discretized the data into equal length bins, and analyzed the discretized data with 5, 10, and 20 bins.

Generating a Bayes net from data first requires one to find an optimal structure. As stated previously, the BN structure is the set of directed links between all variables in the network. Any variable can link to any other variable provided the links are not cyclic. Ideally, one would enumerate and rank all possible structures for a $\mathrm{BN}$, and select the optimal structure based on the rankings.

Unfortunately, the number of possible structures scales super-exponentially with the number of variables, making the previous approach impractical for any BN with more than 4-5 variables. From a recursive formula [8] used to calculate the number of possible structures as function of the number of variables in the BN,

$$
S(n)=\sum_{i=1}^{n}(-1)^{i+1}\left(\begin{array}{l}
n \\
i
\end{array}\right) 2^{i(n-i)} S(n-i),
$$

where $n$ is the number of variables, and $S$ is the number of structures, there are about $8.38 \cdot 10^{46}$ possible BN structures for a network with 16 variables. Therefore, we had to reduce the space of possible structures for the BN.

We used a combination of heuristic algorithms and relevant knowledge about the physics of NIF capsule implosions [9] to help us search for an optimal BN structure. To search the entire dataset for optimal structures, we used a greedy search algorithm [7] to approximate a solution for an optimal structure. The disadvantages of a greedy search technique are that it usually does't find the optimal structure, and that it must depend on the required starting choice.

Relevant knowledge about the physics of NIF implosions was used to limit the space of possible BN networks. The first 4 of the 16 dataset variables (Appendix) were designated as "truth" variables, because these four properties can summarize a capsule implosion with respect to the yield margin [10]. The remaining 12 variables were designated as diagnostics, because they measure output properties of a capsule implosion. Also, unlike the truths, these variables can be directly measured. When 


\begin{tabular}{|c|c|c|c|c|c|}
\hline \multicolumn{7}{|c|}{ Groups of Dataset Variables } \\
\hline Variable \# & Group & Variable \# & Group & Variable \# & Group \\
\hline $\mathbf{1 .}$ & $\mathrm{T}_{0}$ & $\mathbf{5 .}$ & $\mathrm{E}(\mathrm{t})$ & $\mathbf{1 1 .}$ & RC \\
\hline $\mathbf{2 .}$ & $\mathrm{T}_{0}$ & $\mathbf{6 .}$ & $\mathrm{E}(\mathrm{t})$ & $\mathbf{1 2 .}$ & II \\
\hline $\mathbf{3 .}$ & $\mathrm{T}_{0}$ & $\mathbf{7 .}$ & NS & $\mathbf{1 3 .}$ & II \\
\hline $\mathbf{4 .}$ & $\mathrm{T}_{0}$ & $\mathbf{8 .}$ & NS & $\mathbf{1 4 .}$ & II \\
\hline & & $\mathbf{9 .}$ & NS & $\mathbf{1 5 .}$ & NII \\
\hline & & $\mathbf{1 0 .}$ & NS & $\mathbf{1 6 .}$ & NII \\
\hline
\end{tabular}

Table 1: This table shows how the dataset variables were grouped. The first group were the truth variables $\left(\mathrm{T}_{0}\right)$, which dealt with capsule implosion properties. Variables 5-6 dealt with energy produced as a function of time $(\mathrm{E}(\mathrm{t}))$. Variables 7-10 dealt with the neutron energy spectrum (NS). Variable 11 represents the ratio of two radiochemical reactions (RC). Variables 12-14 deal with invasive imaging (II), and the last two variables (15-16) dealt with non-invasive imaging (NII). See the appendix for a more detailed explanation of the variables.

separating the variables into two groups, we assumed that links between variables can only flow from the truths to the diagnostics. This distinction between the truths and diagnostics reduced the number of possible networks from $8.38 \cdot 10^{46}$ to $2.81 \cdot 10^{14}$. We made further reductions to the space of possible BN structures by grouping related variables (Table 1), and exhaustively searching over grouped variables.

\subsection{Mechanisms for Scoring the Structures}

In order to find an optimal BN structure, we needed a mechanism to rank possible BN structures with respect to the data in the dataset. The optimal structure would contain the most likely set of links between the dataset variables based on the dataset. Although there are several mechanisms for scoring BN structures, such as the widely used Bayesian Information Criterion (BIC) [11], we ranked the possible BN structures using a Bayesian scoring method [12], which was based on the BN structures' marginal likelihood, or integrated likelihood.

Marginal likelihood works by integrating the model over all possible parameters. In our case, the model is a possible BN structure, and the parameters are the conditional probabilities for each variable. In order to find an optimal BN structure for the dataset, we had to compute $\mathrm{P}\left(\mathrm{M}_{i} \mid \mathrm{x}\right)$, where $\mathrm{M}_{i}$ is a possible BN structure, and $\mathrm{x}$ is the data in the dataset. (Bayesian statistics allows one to compute the probability of parameters in a model. Conversely, frequentist statistics doesn't allow this; one can only compute the likelihood of a model, and not it's probability. See [13] for a more detailed discussion.) From Bayes' theorem (Eq. 2), we find that

$$
P\left(M_{i} \mid x\right)=\frac{P\left(x \mid M_{i}\right) P\left(M_{i}\right)}{P(x)},
$$


where $\mathrm{M}_{i}$ is a possible $\mathrm{BN}$ structure, $\mathrm{P}\left(\mathrm{x} \mid \mathrm{M}_{i}\right)$ is the probability of observing the data assuming $\mathrm{M}_{i}$ were the correct $\mathrm{BN}$ structure, $\mathrm{P}\left(\mathrm{M}_{i}\right)$ is the probability of $\mathrm{M}_{i}$ being an optimal BN structure independently of the data, and $\mathrm{P}(\mathrm{x})$ is the probability of observing the data. Since we assumed all BN structures were equally likely, we set $\mathrm{P}\left(\mathrm{M}_{i}\right)=\mathrm{P}\left(\mathrm{M}_{j}\right) \forall j$. To compute $\mathrm{P}\left(\mathrm{x} \mid \mathrm{M}_{i}\right)$, we integrate out $\mathrm{M}_{i}$ over all possible conditional probabilities, or

$$
P\left(x \mid M_{i}\right)=\int P\left(x \mid M_{i}, \theta\right) P\left(\theta \mid M_{i}\right) d \theta,
$$

where $\theta$ represents the conditional probabilities for $\mathrm{M}_{i}$. Note that the first term in the integral, $\mathrm{P}\left(\mathrm{x} \mid \mathrm{M}_{i}, \theta\right)$, is a simple frequentist probability - it merely states that given a complete $\mathrm{BN}$, find the probability of observing the data in $\mathrm{x}$. The second term in the integral, $\mathrm{P}\left(\theta \mid \mathrm{M}_{i}\right)$, is the probability of a BN structure having a specific set of probabilities. Since we had no bias towards a particular set of conditional probabilities for a given BN structure, we set this term to have a Dirichlet, or flat top, distribution. (If we did have a bias towards a particlular set of conditional probabilities, we could have used a Gaussian distribution here.)

Computing the $\mathrm{P}(\mathrm{x})$ term (Eq. 9) is difficult, as $\mathrm{P}(\mathrm{x})$ represents the probability of observing the given data. Technically, computing $\mathrm{P}(\mathrm{x})$ would require us to know all possible models that could have generated the data, as

$$
P(x)=\sum_{i} P\left(x \mid M_{i}\right) P\left(M_{i}\right)
$$

However, when comparing the relatively likelihood of one BN structure towards another BN structure, the $\mathrm{P}(\mathrm{x})$ term is cancelled, and we are left with (Eq. 4)

$$
\mathcal{L}\left(M_{i}, M_{j}\right)=\frac{P\left(x \mid M_{i}\right) P\left(M_{i}\right)}{P\left(x \mid M_{j}\right) P\left(M_{j}\right)},
$$

where $\mathscr{L}\left(\mathrm{M}_{i}, \mathrm{M}_{j}\right)$ is the likelihood of $\mathrm{M}_{i}$ relative to $\mathrm{M}_{j}$. With this approach, we could determine the relative likelihood of one BN structure with respect to another BN structure, and find an optimal BN structure for the data.

\subsection{Estimating the Conditional Probabilities}

After finding an optimal structure, one needs to find a set of conditional probabilities for each dataset variable in order to have a complete BN. To find conditional probabilities for a BN based on the dataset and the optimal structure, we used maximum likelihood estimation (MLE). For example, if one has a coin that yields seven heads after ten coin flips, then the probability of the coin landing heads is most likely 0.7. (This concept returns to the discussion in [13] of inverse probability. Also, this 


\begin{tabular}{|c|c|c|}
\hline \multicolumn{3}{|c|}{ Links Between Individual Truth and Diagnostic Variables } \\
\hline Truth \# & Diagnostic \# & Likelihood of a Link ${ }^{*}$ \\
\hline $\mathbf{1 .}$ & $\mathbf{6 .}$ & 2.4 \\
\hline $\mathbf{1 .}$ & $\mathbf{8 .}$ & $6.9 \cdot 10^{3}$ \\
\hline $\mathbf{2 .}$ & $\mathbf{5 .}$ & $4.9 \cdot 10^{5}$ \\
\hline $\mathbf{3 .}$ & $\mathbf{6 .}$ & 12.1 \\
\hline $\mathbf{3 .}$ & $\mathbf{9 .}$ & $2.0 \cdot 10^{4}$ \\
\hline $\mathbf{3 .}$ & $\mathbf{1 2 .}$ & $3.1 \cdot 10^{8}$ \\
\hline $\mathbf{3 .}$ & $\mathbf{1 4 .}$ & 511 \\
\hline \multicolumn{3}{|c|}{ P(No Link Exists | Data) } \\
\hline
\end{tabular}

Table 2: Optimal structures obtained from exhaustively searching the set of all possible individual links between truth and diagnostic variables. We found 7 links out of 48 total possible links. The link between variables $\mathbf{3}$. and 12. was the strongest, whereas the link between variables $\mathbf{1}$. and 6. was the weakest. The equation used for likelihood of a link comes from (Eq. 12).

example is one of the rare MLE examples that can be solved analytically [14].) In our case, if a dataset variable had no parents in our optimal BN structure, then the conditional proababilities for that variable simply reflect its behavior in the dataset. If the dataset variable did have parents, then its conditional probabilities reflect its behavior and its parents' behavior in the dataset. Since our dataset contains 120 simulations for each variable, this method provided us with a good approximation of the conditional probabilities.

\section{Preliminary Results}

\subsection{Finding Optimal Structures}

As stated previously, our dataset contained 16 variables, which leads to $8.38 \cdot 10^{46}$ possible BN structures. In order to find an optimal structure, we either exhaustively searched small subsets of possible BN structures, or used a greedy search algorithm to search larger subsets of BN structures, including the set of all possible BN structures [7]. One method we used to limit the set of possible BN structures is by dividing the variables into truths and diagnostics (Table 1).

The simplest subset of possible BN structures we could search is the subset containing single connections between one truth variable, and one diagnostic variable. Here, we only had to compare two possible BN structures - either there was a link from the truth to the diagnostic variable, or there wasn't. Using eqution 12, we could determine the relative likelihood of whether there was a link between the two variables. Any relative likelihood ratio greater than 1 meant that there was likely 
a link between the two variables. Our results show that at the 5 bin level, there were only seven likely links between individual truth and diagnostic variables. At the 10 and 20 bin levels, there were no likely links between the truth and diagnostic variables.

We then exhaustively searched the subset of BN structures containing links between all 4 truths and 1 diagnostic variable, and the subset of BN structures containing 1 truth and all 12 diagnostic variable. At the 5 bin level searching the subset of BN structures containing links between 4 truths and 1 diagnostic variable yielded all links in Table 2 excluding the link between variables $\mathbf{1}$ and $\mathbf{6}$. Searching this subset at the 10 bin level yielded links between all 4 truth variables and variable $\mathbf{9}$. Searching this subset at the 20 bin level yielded 12 links. These links were between all 4 truths, and variables $\mathbf{5}, \mathbf{9}$, and $\mathbf{1 4}$ (Fig. 3A-C). For the subset of BN structures containing links between 1 truth and all 12 diagnostic variables, at the 5 bin level, we found the seven links in Table 2 (Fig. 3D). At the 10 and 20 bin levels, the optimal structures contained no links (Fig. 3E).

We also exhaustively searched the subset of BN structures containing links between all 4 truths, and the 5 groups of diagnostic variables (Table 1). Some of these subsets were rather large; the largest subset contained $2^{16}=65536$ possible $\mathrm{BN}$ structures. Therefore, in order to reduce the subset of BN structures, we forced the results to contain the six links in Table 2 (all links except the link between variables 1 and 6). At the 5 bin level (Fig. 4A), the optimal structures didn't contain any additional links. Interestingly, at the 10 bin level, our optimal BN structure contained additional links between truth and diagnostic variables. In addition to exhaustively searching small subsets of BN structures, we used greedy search techniques [7] to search over all possible BN structures containing links between truth and diagnostic variables, and the entire dataset. At the 5 bin level, when searching over all possible BN structures containing links between truth and diagnostic variables, our optimal structure only contained the six strongest links from Table 2. When we ignored the division between truth and diagnostic variables, and searched over the entire dataset, our optimal structure contained 7 links between variables (Fig. 5). At the 10 and 20 bin level, our optimal structures obtained from a greedy search algorithm contained no links between any variables. In unpublished work, Brenda $\mathrm{Ng}$ of Lawrence Livermore National Laboratory, has used a more rigorous approach on the same dataset with comparable results.

\subsection{Estimating Conditional Probabilities}

We used a maximum likelihood estimate (MLE) to estimate the conditional probabilities. At the 5 bin level, assume that our optimal BN structure only contains the six strongest links in Table 2 (Fig. 3A). There are two types of variables in this 


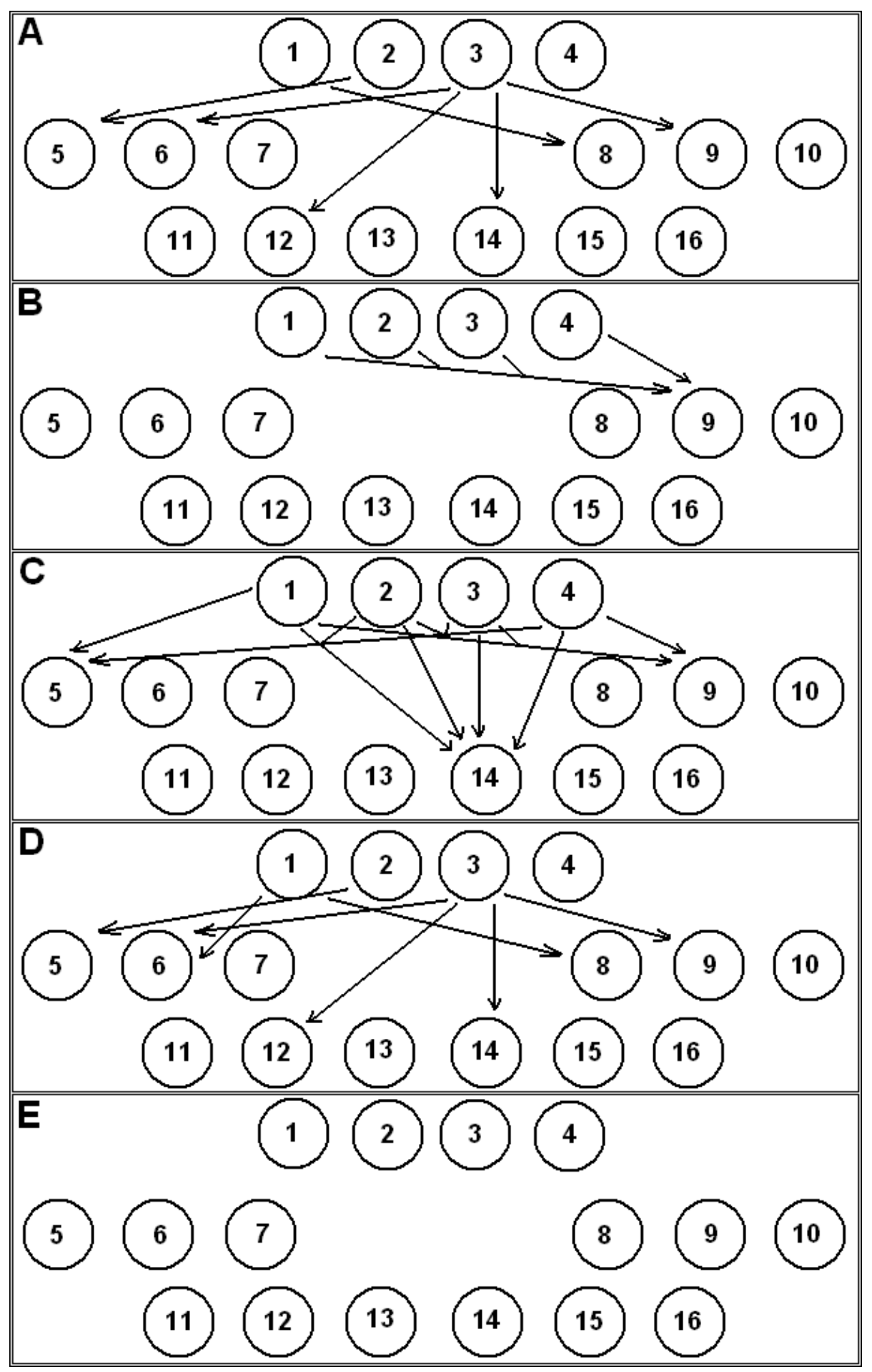

Figure 3: Structures A - C are the optimal structures obtained from searching the subset of BN structures containing links between all 4 truths and 1 diagnostic variable at the 5, 10, and 20 bin level respectively. $\mathbf{D}$ shows the BN structure obtained from searching the subset of BN structures containing links between 1 truth and all 12 diagnostic variables at the 5 bin level, and $\mathbf{E}$ shows the $\mathrm{BN}$ structure from the 10 and 20 bin levels. 


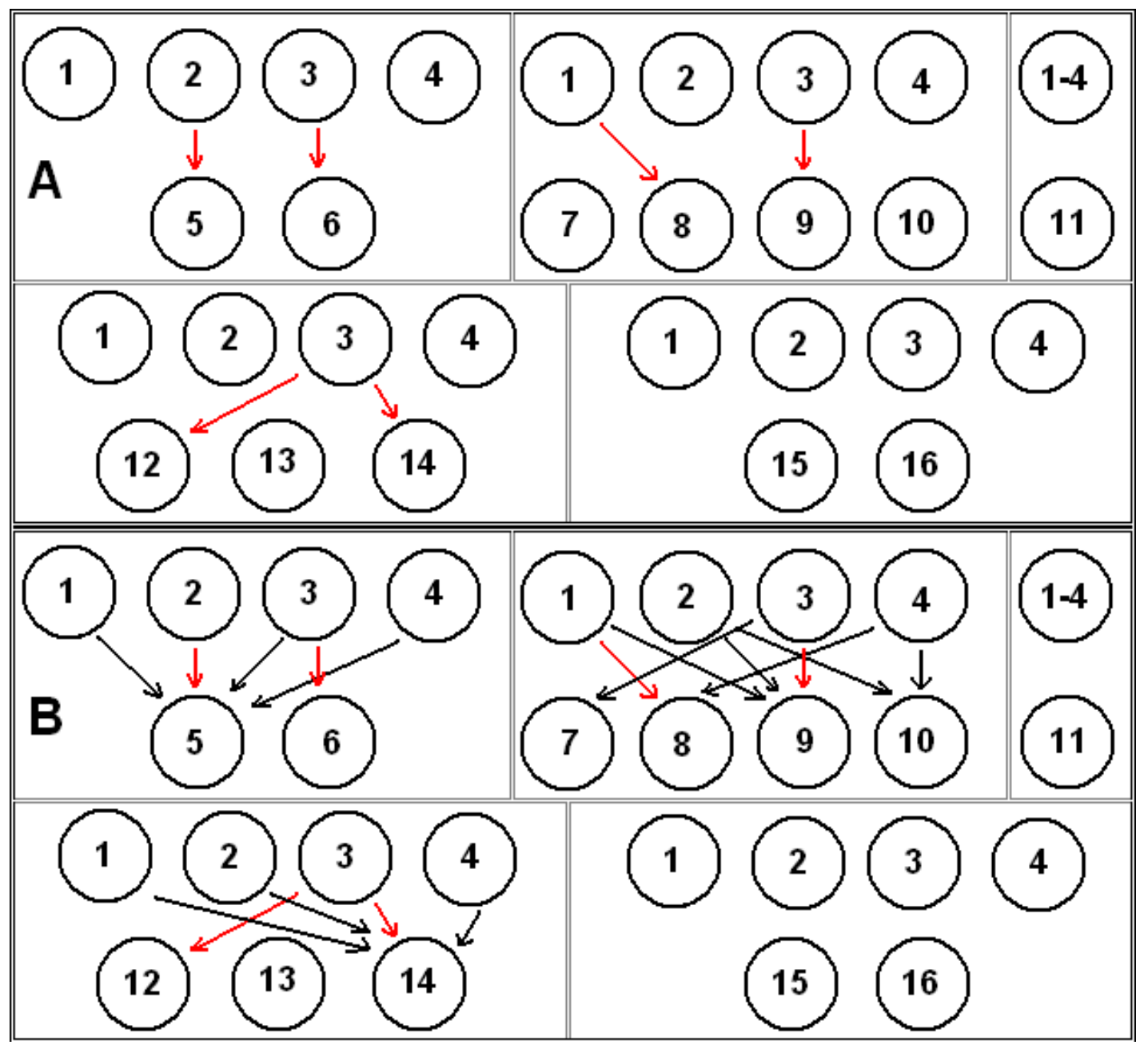

Figure 4: $\mathbf{A}$ and $\mathbf{B}$ contain the structures generated from exhaustively groups of variables at the 5 and 10 bin level respectively. Red arrows represent links that we forced these BN structures to have. Black arrows represent additional links learend when we divided the diagnostic variables into groups. At the 5 bin level (A), the optimal structures only contained the forced links (red arrows). But at the 10 bin level (B), the optimal structures contained additional links. 


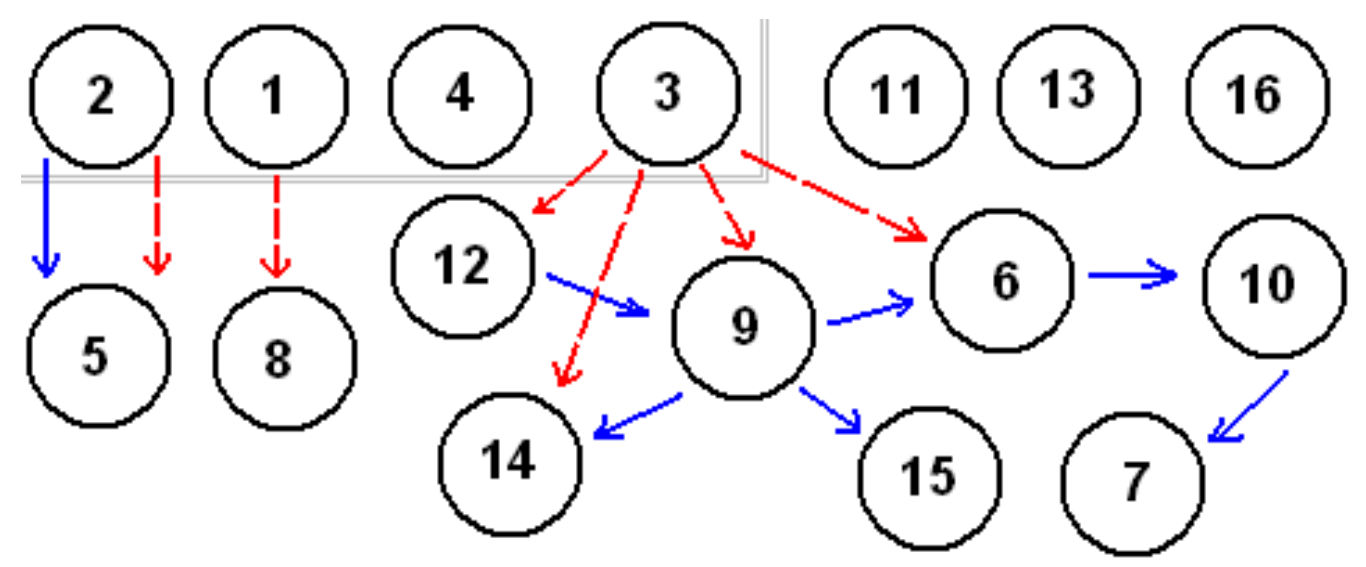

Figure 5: The broken red arrows represent the optimal strucutre generated from a greedy search over all possible links between truth and diagnostic variables. Not coincidentally, these six links are the six strongest links in Table 2. The solid blue arrows represent the optimal structure generated from a greedy search algorithm over the entire dataset.

optimal structure - variables with and without parent variables.

For example, consider the case of variables $\mathbf{2}$ and $\mathbf{5}$ (Fig. 6). Since variable $\mathbf{2}$ has no parents, the MLE of its probabilities is simply its frequency in the dataset. The probability that variable $\mathbf{2}$ is in state $\# 1$ is .14, because in the dataset, variable \#2 is in state \#1 14\% of the time. The MLE of the conditional probabilities for variable $\mathbf{5}$ depends on its frequency and variable $\mathbf{2}$ 's frequency. The probability that variable $\mathbf{5}$ is in state $\# 1$ is .19 if variable $\mathbf{2}$ is in state 4 , and .83 if variable $\mathbf{2}$ is in state 5 . The probability that variable $\mathbf{5}$ is in state $\# 1$ is

$$
P\left(\mathbf{5}=S_{1}\right)=\sum_{i=1}^{5} P\left(\mathbf{5}=S_{1} \mid \mathbf{2}=S_{i}\right) P\left(\mathbf{2}=S_{i}\right)=.19 \cdot .17+.83 \cdot .1=.115,
$$

where $\mathrm{S}_{i}$ is the $i$ 'th state. At the 5 bin level, each variable would have $5^{P}$ conditional probabilities, where $P$ is the number of parents for the variable. Similarly, at the 10 bin level, each variable would have $10^{P}$ conditional probabilities.

\subsection{Explaining Links Between Variables}

As stated previously, we found six strong links between truth and diagnostic variables (Table 2). We can use relevant physics knowledge and the conditional probabilities to explain the link between variables $\mathbf{2}$ and $\mathbf{5}$. Variable $\mathbf{2}$ represents the capsule implosion velocity, and variable 5 represents the time it took the capsule to implode. Intuitively, capsules with higher implosion velocities won't take as long to implode as capsules with lower implosion velocities. The conditional probabilities 
(Fig. 6E) confirm this. If the velocity is in a low state (states $\# 1$ and $\# 2$ ) then imposion time is likely to be in a high state (states \#4 and \#5) and vice versa.

Relevant physics knowledge can also be used to explaining the other five links between truth and diagnostic variables. The strongest link is between variables $\mathbf{3}$ and 12. Variable 3 represents the RMS radius of the hotspot, and variable 12 represents the average radius of the limb obtained from invasive imaging. Similarly, the link between variables $\mathbf{3}$ and $\mathbf{1 4}$, the average collinear density, is due to the fact that a capsule with a larger RMS hotspot radius will have less material outside the hotspot, and a lower collinear density. The link between variables $\mathbf{3}$ and $\mathbf{6}$, the "burn width", or the FWHM of the E(t) (Energy produced vs. time, see Appendix) curve, is also fairly intuitive. A capsule with a larger RMS hotspot radius will have more material burning, and thus, a larger burn width.

The remaining two links are between variables $\mathbf{1}$ (capsule entropy) and $\mathbf{8}$ (fraction of high energy (17-22 MeV) neutrons in the neutron spectrum), and $\mathbf{3}$ and $\mathbf{9}$ (fraction of low energy (10-13 MeV) neutrons in the neutron spectrum). The products of the deuterium-tritium collision are a $3.5 \mathrm{MeV}$ alpha particle, and a $14 \mathrm{MeV}$ neutron, so the neutron spectrum is sharply peaked around $14 \mathrm{MeV}$. Although most of these 14 $\mathrm{MeV}$ neutrons quickly leave the capsule, some of them will scatter off of material, and either lose or gain energy. A reason that variable $\mathbf{1}$ (capsule entropy) is related to variable $\mathbf{8}$ (fraction of high energy neutrons) is that systems with high entropies usually don't have a lot of burn, which means very few $14 \mathrm{MeV}$ neutrons. Since the $14 \mathrm{MeV}$ neutrons can gain energy by scattering off other particles, fewer $14 \mathrm{MeV}$ neutrons results in fewer high energy neutrons. A reason that 3 (RMS hotspot radius) is related to $\mathbf{9}$ (fraction of low energy neutrons) is that a greater hotspot radius means that the neutrons have to travel larger distances to leave the capsule, so more neutrons will lose some energy when leaving the capsule. However the reasons for the links between these variables are not well understood, and need to be investigated further.

\section{Conclusion}

At the 5 bin level after dividing the dataset variables into truth variables and diagnostic variables, most of the structures we found only contained 6 links between the truths and diagnostics. We had expected that there would be more links between the truths and diagnostics. An important reason why we found fewer links than we expected is the relatively small size of the dataset, which only contained 120 simulations. In fact, when we performed a greedy search over the entire dataset, our optimal structure only contained 7 links. Other factors to consider are data binning and sampling. In order to find a link between two variables, we needed a good range of values for each variable. This required the dataset to contain appropriate 
sampling of all sixteen variables. However, some of the dataset variables had very skewed distributions. Since we binned the data using equal length bins, in cases with an extremely skewed distribution of values, binning these variables resulted in almost all the values ending up in one bin. This made it extremely difficult to resolve links between these variables.

There were also physical reasons that we had a small number of links. Although dividing the dataset variables into truths and diagnostics facilitated our search for optimal BN structures, in order to divide the variables into truths and diagnostics, we made several major assumptions. Specifically, we assumed that the truth variables were independent from each other, and that the diagnostics were conditionally independent given the appropriate truth variables. In reality, the truth variables are approximately independent from each other over a certain range of values. For example, if variable $\mathbf{1}$ (entropy) were extremely high, that would limit the range of values for variable $\mathbf{2}$ (implosion velocity). Another factor that influenced our results is the fact that in the dataset, only about $20 \%$ of the simulations represented successful thermonuclear fusion. Successful and unsuccessful simulations are useful in measuring different diagnostic properties of a capsule implosion. Our results might have been different if we were only studying successful or unsuccessful simulations.

In conclusion, with an appropriate dataset, we could construct a complete Bayesian network for modelling capsule implosions. This would allow us to determine the interdependencies of different variables. Specifically, we could determine the actual properties of capsule implosions, which aren't directly measurable, based on the appropriate diagnostics. This allows for uncertainty quantification for NIF capsule implosions.

\section{Acknowledgement}

I would like to thank Steve Libby and Vijay Sonnad for mentoring me for the summer, and helping me with various aspects of this project. I would also like to thank Brian Spears and Brenda Ng for providing me with additional advice. Finally, I would additionally like to thank Brian Spears for generating the dataset used for this project. 


\section{References}

[1] P. Gregory, Bayesian Logical Data Analysis for the Physical Sciences: A Comparative Approach with Mathematica ${ }^{\circledR}$ Support. Cambridge University Press, Cambridge, UK., 2005.

[2] R. E. Neapolitan, Learning Bayesian Networkds. Pearson Prentice Hall, 2004.

[3] F. Jensen and T. Nielsen, Bayesian Networks and Decision Graphs. Springer, 2007.

[4] L. Fu and I. Tsamardinos, "A Comparison of Bayesian Network Learning Algorithms from Continuous Data," AMIA Annual Symposium Proceedings, vol. 2005, p. $960,2005$.

[5] G. Zimmerman and W. Kruer, "Numerical simulation of laser-initiated fusion," Comments Plasma Phys. Controlled Fusion, vol. 2, pp. 51-61, 1975.

[6] K. Murphy et al., "The Bayes Net Toolbox for Matlab," Computing Science and Statistics, vol. 33, pp. 331-350, 2001.

[7] P. Leray and O. Francois, "BNT structure learning package: Documentation and experiments," Reasearch report Laboratoire PSI, INSA Rouen France, 2004.

[8] R. Robinson, "Counting unlabeled acyclic digraphs. Little, CHC (Eds.), Lectures notes in mathematics 622: Combinatorial mathematics V 28-43," 1977.

[9] J. Lindl, "Development of the indirect-drive approach to inertial confinement fusion and the target physics basis for ignition and gain," Physics of Plasmas, vol. 2, p. 3933, 1995.

[10] D. S. Clark, S. W. Haan, and J. D. Salmonson, "Robustness Studies of Ignition Targets for the National Ignition Facility in Two Dimensions," Physics of Plasmas, vol. 15, p. 056305, 2008.

[11] G. Schwarz, "Estimating the dimension of a model," Annals of Statistics, vol. 6, no. 2, pp. 461-464, 1978.

[12] D. Heckerman, D. Geiger, and D. Chickering, "Learning Bayesian networks: The combination of knowledge and statistical data," Machine Learning, vol. 20, no. 3, pp. 197-243, 1995.

[13] R. Fisher, Statistical Methods for Research Workers, pp. 9-11. Hafner Publishing Company, 14th ed., 1970.

[14] I. Myung, "Tutorial on maximum likelihood estimation," Journal of Mathematical Psychology, vol. 47, no. 1, pp. 90-100, 2003. 


\section{Appendix: Dataset Variables}

The dataset analyzed for this project contained sixteen variables. As stated previously, the first four variables are truths, because they can summarize the energy yield of a NIF capsule implosion [10]. These variables are (1.) capsule entropy during implosion, (2.) capsule velocity during implosion, (3.) the RMS radius of the hotspot, and (4.) 1 - the percentage of DT in the shell during implosion. This last variable mainly measures the percentage of ablator that fails to ablate, and gets imploded along with the DT shell.

The remaining twelve variables represent measurable diagnostic properties of a capsule implosion. The first two diagnostic variables are (5.) the time it took the capsule to implode, and (6.) the FWHM of the peak of the burn history of the capsule. The next four variables deal with the neutron spectrum: (7.) the total number of neutrons with energies around $14 \mathrm{MeV}$ produced, the fraction of neutrons with energies between (8.) 17-22 MeV, and (9.) 10-13 MeV, and (10.) the FWHM of the peak of the neutron spectrum (Fig. 7). The next variable is (11.) the ratio of the end products of two radiochemical reactions,

$$
\begin{gathered}
D^{+}+{ }^{127} I \rightarrow{ }^{127} X e+2 n, \\
\alpha^{+}+{ }^{18} \mathrm{O} \rightarrow{ }^{21} \mathrm{Ne}+1 n .
\end{gathered}
$$

The three subsequent variables, (12.) average limb radius, (13.) RMS limb radius $\div$ average limb radius, and (14.) limb $\rho$ r (the collinear density), are obtained from ARC imaging of the limb. The final two variables, (15.) average radius and (16.) RMS radius $\div$ average radius are obtained from self-emission of high energy X-rays. 


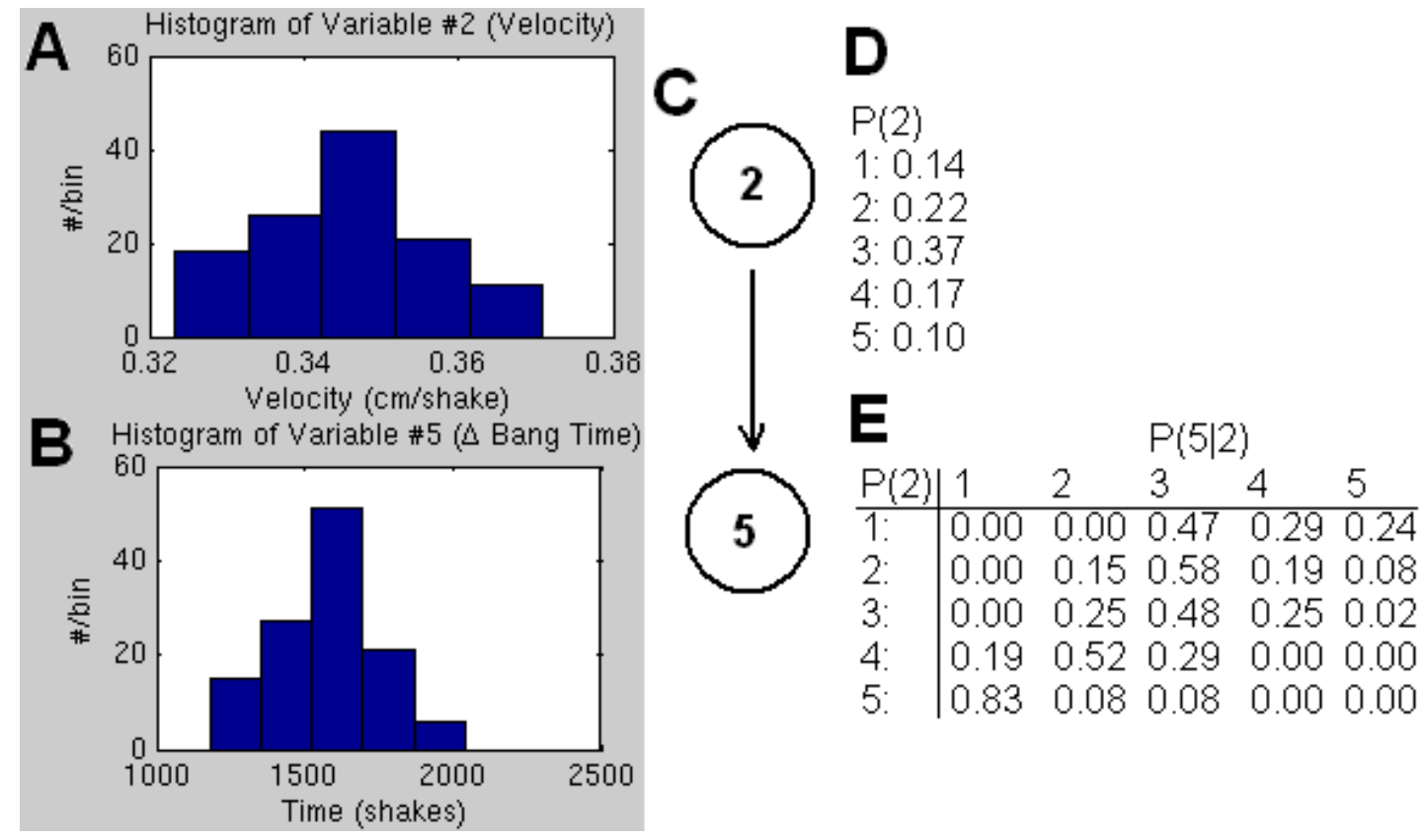

Figure 6: $\mathbf{A}$ and $\mathbf{B}$ are histograms showing the distribution of variables $\mathbf{2}$ and $\mathbf{5}$ at the 5 bin level. $\mathbf{C}$ is the BN structure that shows a directed link between the two variables. $\mathbf{D}$ is the set of probabilities for variable $\mathbf{2}$. $\mathbf{E}$ is the set of conditional probablities of variable $\mathbf{5}$. Variable $\mathbf{5}$ 's probabilities depend on the behavior of variables $\mathbf{2}$ and $\mathbf{5}$. Together, $\mathbf{C}-\mathbf{E}$ represent a complete Bayes net for variables $\mathbf{2}$ and $\mathbf{5}$. 


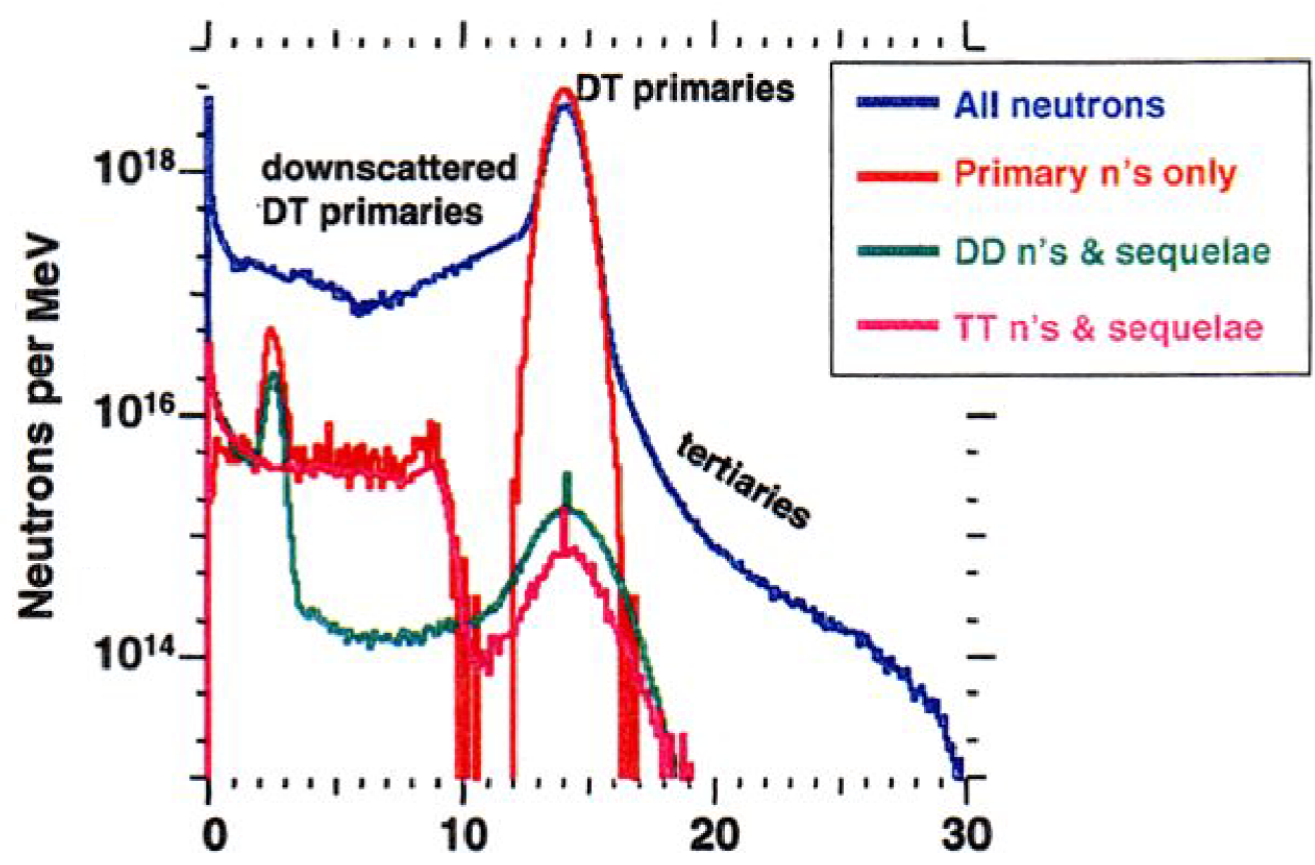

Figure 7: Neutron spectrum used for variables 7-10. Variable $\mathbf{7}$ represents the peak of the spectrum for all neutrons (blue). Variables 8-9 represent the fraction of all neutrons between 10$13 \mathrm{MeV}$ and 17-22 MeV respectively. Variable 10 represents the FWHM of the curve for primary neutrons (orange). 